\title{
A NORM PROPERTY FOR SPACES OF COMPLETELY BOUNDED MAPS BETWEEN C*-ALGEBRAS
}

\author{
by TADASI HURUYA
}

(Received 26 November, 1985)

Let $M_{n}$ be the $\mathrm{C}^{*}$-algebra of $n \times n$ complex matrices. If $A$ is a $\mathrm{C}^{*}$-algebra, let $M_{n}(A)$ denote the $C^{*}$-algebra of $n \times n$ matrices $a=\left[a_{i j}\right]$ with entries in $A$. For a linear map $\phi: A \rightarrow B$ between $C^{*}$-algebras, we define the multiplicity map $\phi_{n}: M_{n}(A) \rightarrow M_{n}(B)$ by $\phi_{n}\left(\left[a_{i j}\right]\right)=\left[\phi\left(a_{i j}\right)\right]$. A linear map $\phi$ is said to be completely bounded if $\sup _{n}\left\|\phi_{n}\right\|<\infty$. Let $B(A, B), \mathrm{CB}(A, B)$ denote the Banach space of bounded linear maps, the set of completely bounded maps from $A$ to $B$, respectively.

A $C^{*}$-algebra $A$ is said to be subhomogeneous if all irreducible representations of $A$ are finite dimensional with bounded dimension. Let $M$ be the von Neumann algebra consisting of all operators which are of the form $a=\sum_{n=1}^{\infty} \oplus a_{n}$, where $a_{n} \in M_{n}$ and $\sup _{n}\left\|a_{n}\right\|<\infty$. Smith proved in [5] that if $A$ is an infinite dimensional $C^{*}$-algebra and $B$ is a $C^{*}$-algebra containing $M$ then the closure of $\mathrm{CB}(A, B)$ in $B(A, B)$, with respect to the operator norm topology, is nowhere dense. He also asked whether this result remains true if $B$ is replaced by a non-subhomogeneous algebra. In this note we shall settle this in the affirmative if $A$ is separable.

A linear map $\phi: A \rightarrow B$ between $C^{*}$-algebras is said to be completely positive if each multiplicity map $\phi_{n}: M_{n}(A) \rightarrow M_{n}(B)$ is positive. If $\phi \in \operatorname{CB}(A, B)$, we put the norm $\|\phi\|_{\mathrm{cb}}=\sup _{n}\left\|\phi_{n}\right\|$. If $\phi$ is completely positive then $\|\phi\|_{\mathrm{cb}}=\|\phi\|$ by Stinespring's theorem $[6$, IV, Theorem 3.6(ii)].

LemMA 1. If a $C^{*}$-algebra $B$ is not subhomogeneous then there exists a sequence $\left\{B_{n}\right\}$ of $C^{*}$-subalgebras of $B$ satisfying the following conditions.

(1) If $n \neq m$ then $a_{n} a_{m}=0$ for any $a_{n}$ in $B_{n}$ and $a_{m}$ in $B_{m}$.

(2) Given any positive number $r>0$, there exist completely positive contractions $\psi_{n}: M_{n} \rightarrow B_{n}$ and $\phi_{n}: B \rightarrow M_{n}$ such that $\left\|\phi_{n} \circ \psi_{n}-\mathrm{id}\right\|<r$ on $M_{n}$ for each $n$.

Proof. For a representation $\pi$ of a $C^{*}$-algebra, let $\operatorname{dim} \pi$ denote the dimension of $\pi$. If a $C^{*}$-subalgebra $B_{0}$ of $B$ admits an irreducible representation $\pi$ with $\operatorname{dim} \pi \geqq m$, there exist completely positive contractions $\psi_{0}: M_{m} \rightarrow B_{0}$ and $\phi_{0}^{\prime}: B_{0} \rightarrow M_{m}$ such that $\left\|\phi_{0}^{\prime} \circ \psi_{0}-\mathrm{id}\right\|<r$ on $M_{m}$ by [5, Lemma 2.7] or [2, Lemma 2], according as $B_{0}$ is unital or nonunital. Since $M_{m}$ is an injective $C^{*}$-algebra, the map $\phi_{0}^{\prime}$ has a completely positive, norm preserving extension $\phi_{0}: B \rightarrow M_{m}$. Hence we show that there exists a sequence $\left\{B_{n}\right\}$ with condition (1) such that each $B_{n}$ admits an irreducible representation $\pi_{n}$ with $\operatorname{dim} \pi_{n} \geqq n$.

(i) We assume first that $B$ has an irreducible representation $\pi$ on an infinite dimensional Hilbert space $H$. Since $\pi(B)$ is infinite dimensional, there exists, by a

Glasgow Math. J. 29 (1987) 181-184. 
standard argument, a self-adjoint element $a$ in $B$ such that the spectrum $Y$ of $\pi(a)$ is infinite (see, for example, the proof of [5, Lemma 2.3]). Then $Y$ is a closed subset of the spectrum $X$ of $a$ by $\left[6, I\right.$, Corollary 8.4]. There then exists a sequence $\left\{f_{n}\right\}$ of positive continuous functions on $X \cup\{0\}$ such that $\left\|f_{n}\right\|=1, f_{n}(0)=0, f_{n}(Y)$ is an infinite set and $f_{n} f_{m}=0$ for $n \neq m$.

Since $f_{n}(a)$ is regarded as an element of $B$, let $B_{n}$ denote the $C^{*}$-subalgebra generated by the algebra $f_{n}(a) B f_{n}(a)$. Then $\left\{B_{n}\right\}$ satisfies condition (1). Let $Q_{n}$ denote the projection of $H$ on the closed subspace generated by $\pi\left(f_{n}(a)\right)(H)$. Since the spectrum of $\pi\left(f_{n}(a)\right)$ contains $f_{n}(Y)$, the space $Q_{n}(H)$ is infinite dimensional. Let $\pi\left(B_{n}\right)^{-}$be the weak closure of $\pi\left(B_{n}\right)$. Then $Q_{n}(H)$ is an invariant subspace under $\pi\left(B_{n}\right)^{-}$. Since $\pi\left(B_{n}\right)^{-}\left|Q_{n}(H)=Q_{n} \pi(B)^{-}\right| Q_{n}(H)=B\left(Q_{n}(H)\right)$, we have the desired irreducible representation $\pi_{n}$ of $B_{n}$ defined by $\pi_{n}(a)=\pi(a) \mid Q_{n}(H)$.

(ii) Suppose that any irreducible representation of $B$ is finite dimensional. Let $B^{\wedge}$ denote the set of primitive ideals of $B$ with the Jacobson topology. Since $B$ is of type 1 , a primitive ideal uniquely corresponds to a unitarily equivalent class of irreducible representations. Also $B$ contains an essential ideal $J$ which has continuous trace by [4, Theorem 6.2.11]. Let $J^{\wedge}$ be the set of primitive ideals $K$ of $B$ such that $K \nsupseteq J$. Then $J^{\wedge}$ is a dense subset of $B^{\wedge}$ as $J$ is essential. By [4, Proposition 4.4.10], there exists a sequence $\left\{\pi_{n}\right\}$ of distinct irreducible representations such that $\operatorname{dim} \pi_{n} \geqq n$ and $K_{n} \in J^{\wedge}$, where $K_{n}$ denotes the kernel of $\pi_{n}$. Since $J$ has continuous trace, $J^{\wedge}$ is a locally compact Hausdorff space by [4, Theorem 6.1.11]. Then there exists a sequence $\left\{f_{n}\right\}$ of positive continuous functions on $B^{\wedge}$ such that the support of $f_{n}$ is contained in $J^{\wedge}, f_{n}\left(K_{n}\right)=1,\left\|f_{n}\right\|=1$ and $f_{n} f_{m}=0$ for $n \neq m$. By Dauns-Hofmann's theorem [4, Corollary 4.4.8], each $f_{n}$ is regarded as an element of the centre of the multiplier of $B$.

Let $B_{n}$ denote the $C^{*}$-subalgebra generated by $f_{n} B$. Then $\left\{B_{n}\right\}$ satisfies condition (1). Since $\pi_{n}\left(f_{n} b\right)=f_{n}\left(K_{n}\right) \pi_{n}(b)=\pi_{n}(b)$ for all $b$ in $B$, the restriction of $\pi_{n}$ to $B_{n}$ is the desired irreducible representation.

Lemma 2. Let $C_{0}(N)$ denote the $C^{*}$-algebra of sequences convergent to 0 . If a $C^{*}$-algebra $B$ is not subhomogeneous then there exists a bounded linear map $\phi: C_{0}(N) \rightarrow B$ such that $\psi$ is not completely bounded whenever $\|\psi-\phi\|<\frac{1}{2}$.

Proof. For a positive integer $n$, let $l_{\infty}(n)$ be the $C^{*}$-algebra of finite sequences $\left\{x_{i}\right\}_{i=1}^{n}$ and put

$$
k(n)=\sup \left\{\|\psi\|_{\mathrm{cb}} \mid \psi: l_{\infty}(n) \rightarrow M_{2} n,\|\psi\| \leqq 1\right\} .
$$

Using Lanford's example, Loebl [3] and Tsui [7] showed that $k(n) \rightarrow \infty$ as $n \rightarrow \infty$; since both $l_{\infty}(n)$ and $M_{2} n$ are finite dimensional, we choose $v_{n}: l_{\infty}(n) \rightarrow M_{2} n$ such that $\left\|v_{n}\right\|_{\mathrm{cb}}=k(n)$ and $\left\|v_{n}\right\|=1$. From Lemma 1 , we have a sequence $\left\{B_{n}\right\}$ of $B$ and completely positive contractions $\psi_{n}: M_{2} n \rightarrow B_{n}$ and $\phi_{n}: B \rightarrow M_{2} n$ such that $\left\|\phi_{n} \circ \psi_{n}-\mathrm{id}\right\|<\frac{1}{2}$ on $M_{2} n$ for each $n$.

Each element $a$ in $C_{0}(N)$ can be written as $a=\sum_{n=1}^{\infty} \oplus a_{n}$, where $a_{n} \in l_{\infty}(n)$ and $\lim _{n}\left\|a_{n}\right\|=0$. If $a=\sum_{n=1}^{\infty} \bigoplus a_{n} \in C_{0}(N)$ then $\left\|\sum_{n=1}^{\infty}\left(\psi_{n} \circ v_{n}\right)\left(a_{n}\right)\right\|=\sup _{n}\left\|\left(\psi_{n} \circ v_{n}\right)\left(a_{n}\right)\right\|$ and 
hence $\sum_{n=1}^{\infty}\left(\psi_{n} \circ v_{n}\right)\left(a_{n}\right)$ belongs to $B$. We define a linear map $\phi: C_{0}(N) \rightarrow B$ by

$$
\phi(a)=\sum_{n=1}^{\infty}\left(\psi_{n} \circ v_{n}\right)\left(a_{n}\right)
$$

Then $\phi$ has norm at most one.

Suppose that there exists a completely bounded map $\psi: C_{0}(N) \rightarrow B$ such that $\|\psi-\phi\|<\frac{1}{2}$. Then

$$
\begin{aligned}
\|\psi\|_{\mathrm{cb}} & \geqq\left\|\phi_{n} \circ \psi \mid l_{\infty}(n)\right\|_{\mathrm{cb}} \\
& \geqq\left\|v_{n}\right\|_{\mathrm{cb}}-\left\|\left(\phi_{n} \circ \psi_{n}-\mathrm{id}\right) \circ v_{n}\right\|_{\mathrm{cb}}-\left\|\phi_{n} \circ(\psi-\phi) \mid l_{\infty}(n)\right\|_{\mathrm{cb}} \\
& \geqq\left(1-\frac{1}{2}-\|\psi-\phi\|\right) k(n) \\
& =\left(\frac{1}{2}-\|\psi-\phi\|\right) k(n) \rightarrow \infty
\end{aligned}
$$

as $n \rightarrow \infty$. This contradicts the fact that $\|\psi\|_{\mathrm{cb}}$ is finite, and completes the proof.

The above lemma is based on an idea of Smith [5, Theorem 2.5].

Theorem 3. Let $A$ be a separable, infinite dimensional $C^{*}$-algebra. If a $C^{*}$-algebra $B$ is not subhomogeneous then there exists a bounded linear map $\Phi: A \rightarrow B$ such that $\psi$ is not completely bounded whenever $\|\psi-\Phi\|<\frac{1}{4}$.

Proof. (i) We first show that there exist completely bounded maps $\phi_{0}: A \rightarrow C_{0}(N)$ and $v_{0}: C_{0}(N) \rightarrow A$ such that $\phi_{0} \circ v_{0}$ is the identity map on $C_{0}(N),\left\|\phi_{0}\right\|_{\mathrm{cb}} \leqq 2$ and $\left\|v_{0}\right\|_{\mathrm{cb}} \leqq 2$.

Let $\alpha N$ denote the one-point compactification of the discrete set of positive integers. If $A$ is unital, by [1, Lemma 5], there exist completely positive unital maps $\phi^{\prime}: A \rightarrow$ $C(\alpha N), v^{\prime}: C(\alpha N) \rightarrow A$ such that $\phi^{\prime} \circ v^{\prime}$ is the identity map on $C(\alpha N)$. Since $C(\alpha N)$ is regarded as $C_{0}(N)+\mathbb{C} I$, we define $\phi_{0}: A \rightarrow C_{0}(N)$ by $\phi_{0}(b)=\phi^{\prime}(b)-f\left(\phi^{\prime}(b)\right) I$, where $f$ denotes a state on $C(\alpha N)$ such that $f(a)=0$ for all $a$ in $C_{0}(N)$, so that $\left\|\phi_{0}\right\|_{\mathrm{cb}} \leqq 2$ and $\phi_{0}(I)=0$. Then $\phi_{0}$ and the restriction $v_{0}$ of $v^{\prime}$ to $C_{0}(N)$ are the desired maps. We remark that $\left\|v_{0}\right\|_{\mathrm{cb}} \leqq 1$.

If $A$ is nonunital, by the above, there exist $\phi^{\prime \prime}: A+\mathbb{C I} \rightarrow C_{0}(N)$ and $v^{\prime \prime}: C_{0}(N) \rightarrow$ $A+\mathbb{C} I$ such that $\phi^{\prime \prime} \circ v^{\prime \prime}$ is the identity map, $\left\|\phi^{\prime \prime}\right\|_{\mathrm{cb}} \leqq 2, \phi^{\prime \prime}(I)=0$ and $\left\|v^{\prime \prime}\right\|_{\mathrm{cb}} \leqq 1$. Let $h$ be a state on $A+\mathbb{C} I$ such that $h(a)=0$ for all $a$ in $A$. We define $v_{0}: C_{0}(N) \rightarrow A$ by $v_{0}(b)=v^{\prime \prime}(b)-h\left(v^{\prime \prime}(b)\right) I$. Then $\left\|v_{0}\right\|_{\mathrm{cb}} \leqq 2$. Since $\phi^{\prime \prime}(I)=0$, the restriction $\phi_{0}=\phi^{\prime \prime} \mid A$ and $v_{0}$ are the desired maps.

(ii) Let $\phi$ be the map as in Lemma 2. Using $\phi_{0}$ and $v_{0}$ as in (i), we put $\Phi=\phi \circ \phi_{0}: A \rightarrow B$. Suppose that there exists a completely bounded map $\psi: A \rightarrow B$ with $\|\psi-\Phi\|<\frac{1}{4}$. Then $\psi \circ v_{0}: C_{0}(N) \rightarrow B$ is a completely bounded map with

$$
\left\|\psi \circ v_{0}-\phi\right\|=\left\|\psi \circ v_{0}-\Phi \circ v_{0}\right\| \leqq\|\psi-\Phi\|\left\|v_{0}\right\|<\frac{1}{2}
$$

By Lemma 2, $\psi \circ v_{0}$ is not completely bounded. This is a contradiction, and completes the proof. 


\section{REFERENCES}

1. T. Huruya, Decompositions of completely bounded maps, Acta Sci. Math. (Szeged), 50 (1986), 183-189.

2. T. Huruya and J. Tomiyama, Completely bounded maps of $\mathrm{C}^{*}$-algebras, J. Operator Theory 10 (1983), 141-152.

3. R. I. Loebl, A Hahn decomposition for linear maps, Pacific J. Math. 65 (1976), 119-133.

4. G. K. Pedersen, $C^{*}$-algebras and their automorphism groups (Academic Press, 1979)

5. R. R. Smith, Completely bounded maps between $C^{*}$-algebras, J. London Math. Soc. (2) 27 (1983), 157-166.

6. M. Takesaki, Theory of operator algebras I (Springer, 1979).

7. S.-K. J. Tsui, Decompositions of linear maps, Trans. Amer. Math. Soc. 230 (1977), 87-112.

Faculty of Education

NiIgATA UnIVERSITY

NiIgata 950-21, JaPAN 